\title{
CrystEngComm
}

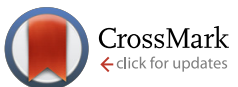

Cite this: CrystEngComm, 2016, 18, 6149

Received 24th May 2016, Accepted 12th July 2016

DOI: $10.1039 / c 6 c e 01200 k$

\section{Structural and electronic characterisation of $\pi$-extended tetrathiafulvalene derivatives as active components in field-effect transistors $\uparrow$}

\author{
Antonio Campos, ${ }^{a}$ Neil Oxtoby, ${ }^{a}$ Sergi Galindo, ${ }^{a}$ Raphael Pfattner, ${ }^{a}$ Jaume Veciana, ${ }^{a}$ \\ Stefan T. Bromley, ${ }^{\text {bc }}$ Concepció Rovira*a and Marta Mas-Torrent ${ }^{\star a}$
}

www.rsc.org/crystengcomm

The electronic and structural properties of two tetrathiafulvalene derivatives bearing aromatic benzene rings are reported. Thin film transistors of these materials show p-type characteristics with comparable mobility values. It is found that the rigidification of the molecule is beneficial for reducing the reorganisation energy but also has an unfavorable impact on the electronic structure dimensionality.

Organic semiconductors have attracted a lot of attention in the last two decades because of their high potential as active components in low cost electronics such as RFID tags, sensors or displays. ${ }^{1}$ Further, organic materials offer the possibility that by chemically modifying their molecular cores their electronic and structural characteristics can be tuned.

In particular, the fast development of organic field-effect transistors (OFETs) has resulted in devices exhibiting mobilities equalling or even surpassing that of amorphous silicon. ${ }^{2,3}$ One of the main pillars for progressing in this field is thus the design of novel semiconductors in order to gain further insights into the structural or electronic molecular properties required for achieving high device performance. ${ }^{4}$ It is generally agreed that the transport mechanism in organic semiconductors is dominated by the hopping transport. According to Marcus theory, ${ }^{5}$ the efficiency of hopping conduction is determined by the electronic intermolecular interactions evaluated through the transfer integral $(t)$, which needs to be maximised, and the reorganisation energy $(\lambda)$

\footnotetext{
${ }^{a}$ Institut de Ciència de Materials de Barcelona (ICMAB-CSIC), and Networking Research Center on Bioengineering, Biomaterials and Nanomedicine (CIBER-BBN), Campus Universitari de Bellaterra, Cerdanyola, E-08193 Barcelona, Spain.

E-mail: cun@icmab.es, mmas@icmab.es; Fax: +34 935805 729;

Tel: +34935801853

${ }^{b}$ Departament de Ciència de Materials i Física Química \& Institut de Química Teòrica i Computacional (IQTCUB), Universitat de Barcelona, E-08028 Barcelona, Spain ${ }^{c}$ Institució Catalana de Recerca i Estudis Avançats (ICREA), E-08010 Barcelona, Spain $\dagger$ Electronic supplementary information (ESI) available: Experimental procedures, characterization data, XRD single crystal data, computational details and device fabrication. CCDC 1460868 and 1460869. For ESI and crystallographic data in CIF or other electronic format see DOI: 10.1039/c6ce01200k
}

which has to be minimised. Typically, more planar and rigid molecular systems will give rise to lower $\lambda$ values since the molecular geometry of the charged and neutral molecule will be more similar. ${ }^{6,7}$ On the other hand, the transfer integral is a variable which generally depends on the relative positions and orientations of neighbouring molecules and the shapes of their frontier orbitals. ${ }^{8}$ It is expected though that the extension of the $\pi$-conjugation improves the intermolecular electronic interactions and, thus, it is beneficial to charge transport. Therefore, the design of organic semiconductors with fused $\pi$-extended systems would, in principle, lead to low $\lambda$ and high $t$ values and hence provide a promising route to achieve high mobility OFETs. ${ }^{9}$ However, in order to realise molecular-performance correlation studies, it is imperative to perform systematic studies carrying out controlled molecular modifications in the organic semiconductor backbone. Tetrathiafulvalene (TTF) derivatives have been shown to be excellent candidates for organic semiconductors in OFETs. ${ }^{10,11}$ TTFs are good electron donor molecules that show a high tendency to form ordered stacks or two-dimensional sheets, which are stabilized by both intermolecular $\pi-\pi$ and $S \cdots S$ interactions. This family of molecules is generally soluble in conventional organic solvents and can be subjected to a large number of synthetic transformations, a key aspect for the design of customized active materials for potential devices. ${ }^{12}$

In this paper we report an experimental and theoretical study of two TTF derivatives, namely bis(4,5-dihydronaphto$[1,2-d])$ tetrathiafulvalene (BDHN-TTF) and bis(naphtho[1,2d])tetrathiafulvalene (BN-TTF) as organic semiconductors in OFETs (Scheme 1). Both molecules mainly differ in the

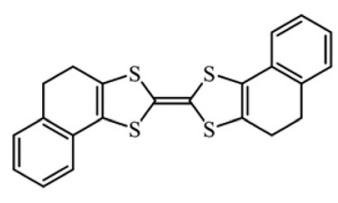

BDHN-TTF

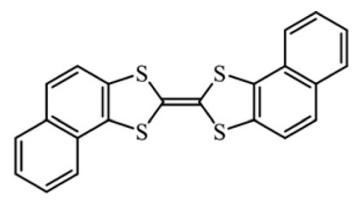

BN-TTF
Scheme 1 
extension of the $\pi$-system in the TTF core. It is observed that both materials reveal a p-type semiconductor behaviour. Although the device performance is rationalized in terms of reorganisation energy and transfer integral, it is clearly elucidated that the electronic dimensionality also plays a crucial role here.

BDHN-TTF was purchased from Aldrich and BN-TTF was synthesized by oxidation of BDHN-TTF with 2,3-dichloro-5,6dicyano-1,4-benzoquinone (DDQ) in dry toluene under an inert atmosphere refluxing for 20 hours. After recrystallisation in toluene, BN-TTF was obtained in $61 \%$ yield (see the ESI $\dagger$ ).

The electronic properties of the two TTF derivatives were investigated by cyclic voltammetry (CV) using $\mathrm{Ag} / \mathrm{AgCl}$ as the reference electrode, a platinum wire as the working and counter electrode and a $0.1 \mathrm{M}$ solution of tetrabutylammonium hexafluorophosphate in $\mathrm{CH}_{2} \mathrm{Cl}_{2}$ as the electrolyte. In the voltammogram of BDHN-TTF and BN-TTF, two reversible oxidation peaks were observed, attributed to the sequential oxidation of the TTF to the radical cation species and the dication (Table 1). It is noticed that the oxidation of BN-TTF takes place at a higher voltage than that of BDHN-TTF, in agreement with a lower lying highest occupied molecular orbital (HOMO). The HOMO energy levels were experimentally estimated from the first oxidation peak (Table 1) according to: $E_{\text {Hомо }}=-\left[E_{\text {onset }}^{\text {ox1 }}+4.7\right] \mathrm{eV}$ (ref. 13 and 14) (Table 1). Also, UV-vis-NIR spectra were recorded for both materials in $\mathrm{CH}_{2} \mathrm{Cl}_{2}$ where the onset of the least energetic band was used to calculate the energy gap between the HOMO and the lowest unoccupied molecular orbital (LUMO).

The electronic characteristics of BDHN-TTF and BN-TTF were also calculated by density functional theory (DFT) using the B3LYP functional ${ }^{15}$ and a $6-311 G+(d, p)$ basis set. All reported DFT calculations were carried out with the Gaussian09 code. $^{16}$ As can be seen in Table 1 , the experimentally obtained $E_{\mathrm{g}}^{\text {opt }}$ and $E_{\mathrm{HOMO}}^{\exp }$ values by UV-vis-NIR spectroscopy and $\mathrm{CV}$, respectively, are in reasonable agreement with $E_{\mathrm{g}}^{\text {calc }}$ and $E_{\text {HOMO }}^{\text {calc }}$. Although the calculated absolute values of $E_{\mathrm{g}}$ and $E_{\text {HOMO }}$ tend to be $\sim 0.4-0.5 \mathrm{eV}$ higher than experimental

Table 1 Redox potentials (versus $\mathrm{Ag} / \mathrm{AgCl}$ ), lowest energy absorption band $\left(E_{\max }\right)$ and optical band gap $\left(E_{\mathrm{g}}^{\text {opt }}\right)$ as well as the experimental and calculated HOMO and LUMO energies ( $E_{\text {HOMO }}$ and $E_{\text {LUMO }}$ ) and calculated HOMO-LUMO gaps (E $\left.E_{g}^{\text {calc }}\right)$ of compounds BDHN-TTF and BN-TTF

\begin{tabular}{lllll}
\hline Compound & & BDHN-TTF & BN-TTF & $\Delta E$ \\
\hline Electrochemical data & $E_{1 / 2}^{\text {ox1 }}[\mathrm{V}]$ & 0.44 & 0.64 & +0.20 \\
& $E_{1 / 2}^{\text {ox2 }}[\mathrm{V}]$ & 0.92 & 1.13 & +0.21 \\
& $E_{\mathrm{On} 1}^{\text {oxset }}[\mathrm{V}]$ & 0.33 & 0.51 & +0.18 \\
& $E_{\mathrm{HOMO}}^{\text {exp }}[\mathrm{eV}]^{a}$ & -5.03 & -5.21 & +0.18 \\
UV-vis data & $E_{\max }\left[\mathrm{cm}^{-1}\right]$ & 21740 & 21410 & -320 \\
& $E_{\mathrm{g}}^{\text {opt }}[\mathrm{eV}]^{b}$ & 2.70 & 2.65 & -0.05 \\
DFT calculations & $E_{\mathrm{HOMO}}^{\text {calc }}[\mathrm{eV}]$ & -4.63 & -4.86 & +0.23 \\
& $E_{\mathrm{LUMO}}^{\text {calc }}[\mathrm{eV}]$ & -1.37 & -1.63 & +0.26 \\
& $E_{\mathrm{g}}^{\text {calc }}[\mathrm{eV}]$ & 3.26 & 3.23 & -0.03
\end{tabular}

${ }^{a}$ Estimated from the onset oxidation potential of the first oxidation peak using the empirical equation: $E_{\text {Hомо }}=-\left[E_{\text {onset }}^{\text {ox1 }}+4.7\right] \mathrm{eV}$. ${ }^{b}$ Determined from the onset of the lowest energy electronic absorption band in the UV-vis spectrum in $\mathrm{CH}_{2} \mathrm{Cl}_{2}$. estimates, the corresponding differences in these energies between the two compounds match very well, in line with our previous studies. $^{17}$

High quality orange block-shaped single crystals of BDHNTTF were grown by slow evaporation of a solution of the molecule in $p$-xylene. BDHN-TTF crystallized in a solvate-free form in the monoclinic space group $P 2_{1} / c$. The crystal structure contains half of a molecule in the asymmetric unit cell. Each TTF molecule is clearly bended in a chair-like conformation (Fig. 1a). The angle between the planes formed by the TTF core and the external benzene ring is $19.3^{\circ}$. BDHN-TTF crystallises forming layers of molecules in the $b c$ plane following a herringbone fashion (i.e., edge-to-face orientation) as can be observed in Fig. 1b. Within these sheets, there are short edge-to-edge-S $\cdots$ S contacts (3.619 $\AA$ ) along $c$.

Yellow needle-shaped single crystals of BN-TTF were grown by diffusion of diethyl ether into a solution of the molecule in chlorobenzene. In this case, the molecule crystallizes in the triclinic space group $P \overline{1}$ and the asymmetric unit cell also consists of half of a BN-TTF molecule, hence, each naphthalene unit is symmetrically equivalent. In the case of $\mathrm{BN}$ TTF, the molecules are very flat, as expected due to its extended aromatic molecular structure (Fig. 2a). The molecules stack in columns along the $a$ axis slightly shifted along the long molecular axis, with an interplanar spacing of $3.574 \AA$
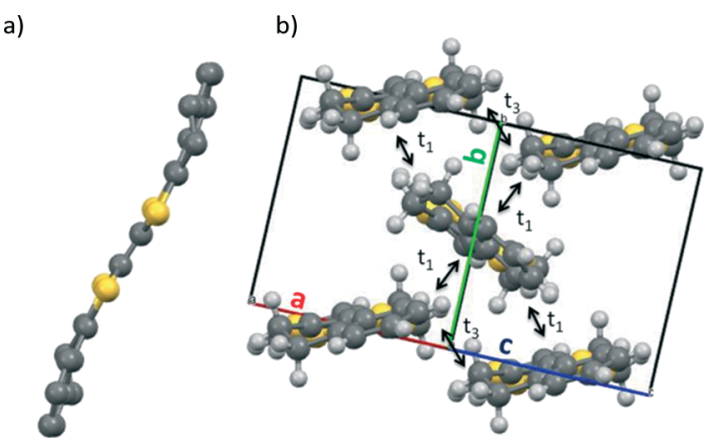

Fig. 1 (a) Non-planar BDHN-TTF molecule found in the crystal structure. $\mathrm{H}$ atoms are omitted. (b) BDHN-TTF packing following a 2-D herringbone network. The $t_{1}$ and $t_{3}$ arrows indicate the directions where the transfer integrals have been calculated. a)

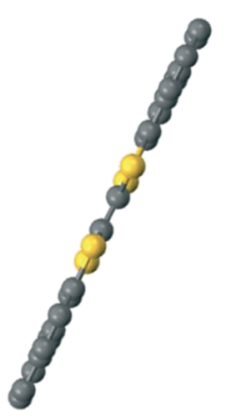

b)

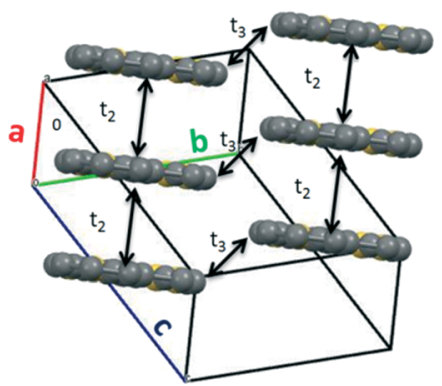

Fig. 2 (a) Planar BN-TTF molecule found in the crystal structure. (b) Displaced cofacial packing of BN-TTF showing the calculated electronic couplings $\left(t_{2}\right.$ and $\left.t_{3}\right) . \mathrm{H}$ atoms are omitted for clarity. 
(Fig. 2b). Short $S \cdots S$ contacts $(3.927 \AA$ ) are also found within the stack and between stacks (3.642 $\AA$ ).

Dimers of BDHN-TTF and BN-TTF taken from the resolved crystal structures were used to calculate their hole transfer integrals $(t)$. As recommended in ref. 18, DFT calculations using the PW91 functional ${ }^{19}$ were conducted to calculate the HOMO energy splitting of the dimers ( $t$ for hole conduction is defined as half of the HOMO energy splitting). The calculated values show that a significant electronic interaction is found for the edge-to-face orientation for BDHN-TTF, with a minor edge-to-edge component. In BN-TTF, however, the preferential transport direction is face-to-face, suggesting a strong 1D favoured electronic path (Table 2). Furthermore, the calculated $t$ values predict a slightly stronger intermolecular electronic coupling in BDHN-TTF crystals (109 $\mathrm{meV}$ ) than in BN-TTF (97 meV). On the other hand, the reorganisation energy, calculated at the B3LYP/6-311+G(d,p) level of theory, is only slightly lower in BN-TTF due to its more planar and rigid structure.

OFET devices were fabricated on a bottom-gate bottomcontact configuration using $\mathrm{Si} / \mathrm{SiO}_{2}$ substrates modified with a self-assembled monolayer of octadecyltrichlorosilane (OTS) with an interdigitated $\mathrm{Cr} / \mathrm{Au}$ source and drain electrodes $(W=$ $25 \mathrm{~mm}$ and $L=25 \mu \mathrm{m})$. The semiconductor was deposited by thermal evaporation $\left(P=9 \times 10^{-7} \mathrm{mbar}\right.$ and rate $\left.=0.2-0.3 \AA \mathrm{s}^{-1}\right)$.

The evaporated thin films were first characterized by X-ray powder diffraction (XRD) and Atomic Force Microscopy (AFM) (Fig. 3). The diffractogram of BDHN-TTF shows clear equidistant diffraction peaks indicative of the crystallinity of the film as well as the high orientation of the crystals. By comparison with the single crystal structure resolved, it is possible to conclude that both the thin film and the single crystal belong to the same phase. The peaks correspond to the family of (noo) reflections and thus, the crystals are oriented with the $b c$ plane parallel to the surface, that is, the plane where significant electronic interactions exist (Fig. 3b, left). Accordingly, the AFM images show that the BDHN-TTF films are formed by $2 \mathrm{D}$ plate-like structures (Fig. 3a, left).

The XRD pattern of BN-TTF shows only two peaks that seem to correspond to the (001) and (010) diffractions of the reported single crystal phase (Fig. 3b, right). The AFM images seem to confirm that BN-TTF prefers to form more 1D tapelike structures (Fig. 3a, right). These results point out that the crystals grow with the $a$ axis parallel to the surface (i.e., the longest crystal direction of BN-TTF crystals), which corresponds to the stacking direction, which is beneficial for the OFET measurements.

Table 2 BDHN-TTF and BN-TTF reorganisation energies $(\lambda)$ and most significant $\mathrm{HOMO}$ transfer integrals $(t)$

\begin{tabular}{lllll}
\hline & $\begin{array}{l}\lambda \\
(\mathrm{eV})\end{array}$ & $\begin{array}{l}\text { Edge-to-face, } \\
t_{1}(\mathrm{meV})\end{array}$ & $\begin{array}{l}\text { Face-to-face } \\
t_{2}(\mathrm{meV})\end{array}$ & $\begin{array}{l}\text { Edge-to-edge } \\
t_{3}(\mathrm{meV})\end{array}$ \\
\hline BDHN-TTF & 0.235 & 109 & - & 29 \\
BN-TTF & 0.219 & - & 97 & 2
\end{tabular}

a)
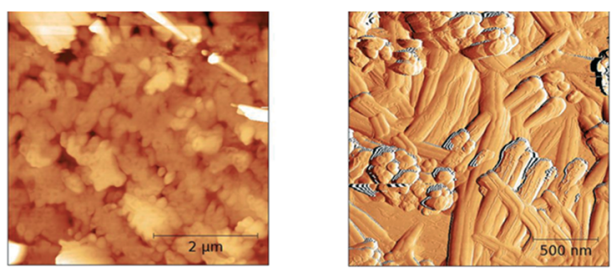

b)
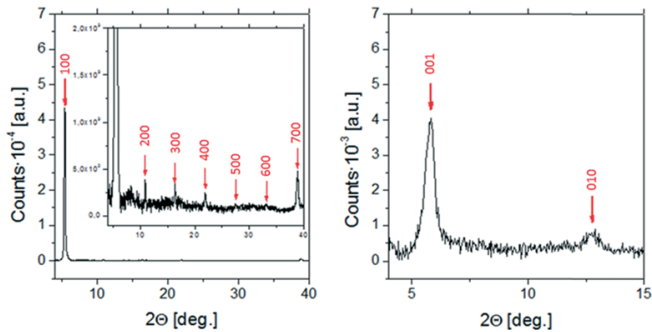

Fig. 3 AFM images (a) and X-ray powder diffraction (b) of the evaporated thin films of BDHN-TTF (left) and BN-TTF (right).

Electrical measurements of the devices were carried out inside a glove box with levels of $\mathrm{O}_{2}$ and $\mathrm{H}_{2} \mathrm{O}$ below $1 \mathrm{ppm}$ to avoid the oxidation of the TTFs. Both materials behave as p-type semiconductors, that is, holes are accumulated in the channel by application of a negative gate voltage. BDHN-TTF OFETs exhibit a mobility of $1 \times 10^{-3} \mathrm{~cm}^{2} \mathrm{~V}^{-1} \mathrm{~s}^{-1}$ with a threshold voltage $\left(V_{\mathrm{TH}}\right)$ of $-11 \mathrm{~V}$ and low hysteresis (Fig. 4a). Some contact resistance can be found in the devices probably because of the concentration of defects on the gold-organic semiconductor interface. ${ }^{20}$ BN-TTF OFETs have a mobility of $2 \times 10^{-3} \mathrm{~cm}^{2} \mathrm{~V}^{-1} \mathrm{~s}^{-1}$ with a large positive $V_{\mathrm{TH}}=33 \mathrm{~V}$ after a thermal annealing at $100{ }^{\circ} \mathrm{C}$ for one hour (Fig. 4b). Such a large positive threshold voltage has also been typically found for the parent material dibenzo-tetrathiafulvalene (DB-TTF). ${ }^{21-25}$ From the electrical characterisation, it can be concluded that
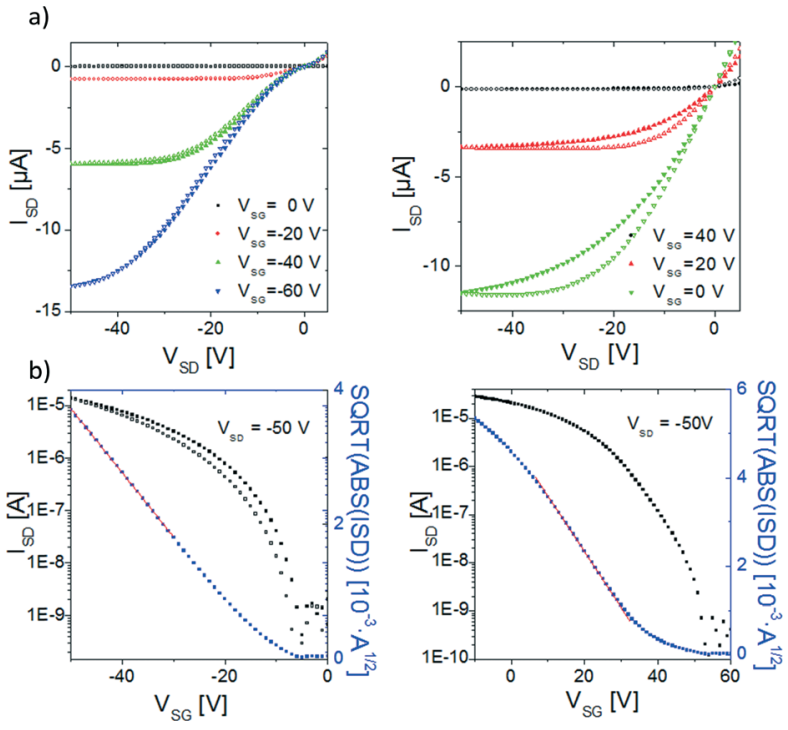

Fig. 4 Typical output (a) and transfer (b) characteristics of BDHN-TTF (left) and BN-TTF (right). 
both materials show similar mobility values. We believe that the different electronic dimensionality of the two materials plays a crucial role. The two dimensional character of BDHN-TTF crystals leads to efficient edge-to-face electronic intermolecular interactions and field-effect mobilities comparable to the ones found in BN-TTF.

\section{Conclusions}

In conclusion, we have successfully characterized the crystal structure of BDHN-TTF and BN-TTF as well as their electronic properties experimentally and theoretically. The reorganization energy values estimated for BN-TTF are reduced with respect to BDHN-TTF revealing that the extension of the $\pi$-system should be beneficial for charge transport. However, it is observed that the molecular modifications also have a strong impact on the crystal structure. BDHN-TTF crystallises in a herringbone pattern exhibiting significant intermolecular interactions in the $b c$ plane, whilst BN-TTF shows a 1-D cofacial packing. Thin film OFET devices were fabricated with these materials. Both materials exhibited similar device performance which was accounted for by the balance between the calculated reorganisation energy values and the electronic dimensionality. Thus, we demonstrate that molecular engineering is important in order to obtain high mobility materials; however, the crystal structure, which is harder to predict, and, specifically, the electronic dimensionality also play crucial roles.

\section{Acknowledgements}

This work was funded by the ERC StG 2012-306826 e-GAMES project, the Networking Research Center on Bioengineering, Biomaterials and Nanomedicine (CIBER-BBN), the DGI (Spain) with project BE-WELL CTQ2013-40480-R and CTQ2015-64618-R, Generalitat de Catalunya 2014-SGR-17, 2014SGR97 and XRQTC and Severo Ochoa Programme for Centres of Excellence in R\&D (SEV-2015-0496). A. C. is enrolled in the Materials Science PhD program of UAB. We acknowledge support of the publication fee by the CSIC Open Access Publication Support Initiative through its Unit of Information Resources for Research (URICI).

\section{Notes and references}

1 G. Gelinck, P. Heremans, K. Nomoto and T. D. Anthopoulos, Adv. Mater., 2010, 22, 3778-3798.

2 M. Mas-Torrent and C. Rovira, Chem. Soc. Rev., 2008, 37, 827-838.

3 H. Sirringhaus, Adv. Mater., 2014, 26, 1319-1335.

4 M. Mas-Torrent and C. Rovira, Chem. Rev., 2011, 111, 4833-4856.
5 R. A. Marcus, Angew. Chem., Int. Ed. Engl., 1993, 32, 1111-1121.

6 S. T. Bromley, M. Mas-Torrent, P. Hadley and C. Rovira, J. Am. Chem. Soc., 2004, 126, 6544-6545.

7 V. Coropceanu, J. Cornil, D. A. da Silva Filho, Y. Olivier, R. Silbey and J.-L. Brédas, Chem. Rev., 2007, 107, 926-952.

8 J. L. Brédas, D. Beljonne, V. Coropceanu and J. Cornil, Chem. Rev., 2004, 104, 4971-5003.

9 W. Wu, Y. Liu and D. Zhu, Chem. Soc. Rev., 2010, 39, 1489-1502.

10 H. Jiang, X. Yang, Z. Cui, Y. Liu, H. Li, W. Hu and C. Kloc, CrystEngComm, 2014, 16, 5968.

11 M. Mas-Torrent and C. Rovira, J. Mater. Chem., 2006, 16, 433.

12 R. Pfattner, S. T. Bromley, C. Rovira and M. Mas-Torrent, Adv. Funct. Mater., 2015, 1-20.

13 T. Lee, C. A. Landis, B. M. Dhar, B. J. Jung, J. Sun, A. Sarjeant, H. J. Lee and H. E. Katz, J. Am. Chem. Soc., 2009, 131, 1692-1705.

14 A. J. Bard and L. R. Faulkner, Electrochem. Methods, 2nd edn, Wiley, New York, 2001.

15 F. J. Devlin, J. W. Finley, P. J. Stephens and M. J. Frisch, J. Phys. Chem., 1995, 99, 16883-16902.

16 D. J. Frisch, M. J. Trucks, G. W. Schlegel, H. B. Scuseria, G. E. Robb, M. A. Cheeseman, J. R. Scalmani, G. Barone, V. Mennucci, B. Petersson, G. A. Nakatsuji, H. Caricato, M. Li, X. Hratchian, H. P. Izmaylov, A. F. Bloino, J. Zheng and G. Sonnenber, Gaussian, Inc., Wallingford CT, 2009, pp. 2-3.

17 Y. Geng, R. Pfattner, A. Campos, W. Wang, O. Jeannin, J. Hauser, J. Puigdollers, S. T. Bromley, S. Decurtins, J. Veciana, C. Rovira, M. Mas-Torrent and S. X. Liu, Chemistry, 2014, 20, 16672-16679.

18 J. Huang and M. Kertesz, J. Chem. Phys., 2005, 122, 234707.

19 J. Perdew, J. Chevary, S. Vosko, K. Jackson, M. Pederson, D. Singh and C. Fiolhais, Phys. Rev. B: Condens. Matter Mater. Phys., 1993, 48, 4978.

20 C. Liu, Y. Xu and Y. Y. Noh, Mater. Today, 2015, 18, 79-96.

21 C. Goh, R. J. Kline, M. D. McGehee, E. N. Kadnikova and J. M. J. Fŕchet, Appl. Phys. Lett., 2005, 86, 1-3.

22 B. Noda, H. Wada, K. Shibata, T. Yoshino, M. Katsuhara, I. Aoyagi, T. Mori, T. Taguchi, T. Kambayashi, K. Ishikawa and H. Takezoe, Nanotechnology, 2007, 18, 424009.

23 J. Nagakubo, M. Ashizawa, T. Kawamoto, A. Tanioka and T. Mori, Phys. Chem. Chem. Phys., 2011, 13, 14370-14377.

24 T. Yamada, T. Hasegawa, M. Hiraoka, H. Matsui, Y. Tokura and G. Saito, Appl. Phys. Lett., 2008, 92, 3-6.

25 Naraso, J. Nishida, S. Ando, J. Yamaguchi, K. Itaka, H. Koinuma, H. Tada, S. Tokito and Y. Yamashita, J. Am. Chem. Soc., 2005, 127, 10142-10143. 